\title{
Microwave Transmission through a Single Subwavelength Annular Aperture in a Metal Plate
}

\author{
Matthew J. Lockyear, Alastair P. Hibbins, and J. Roy Sambles \\ School of Physics, University of Exeter, Exeter EX4 4QL, United Kingdom \\ Christopher R. Lawrence \\ QinetiQ Limited, Cody Technology Park, Ively Road, Farnborough GU14 OLX, United Kingdom
}

(Received 2 February 2005; published 17 May 2005)

\begin{abstract}
The resonant transmission of a small annular aperture, with a diameter much smaller than the radiation wavelength, in a thin metal plate is studied at microwave frequencies. It transpires that such an annular aperture supports several resonant guided modes, including those that are not quantized in the azimuthal direction. Such modes have resonant frequencies that are largely independent of the diameter of the annular aperture, thus being supported by annular apertures that tend to zero radius. The transmittance of such a structure at microwave frequencies is detailed and compared with the predictions of a finite element method model.
\end{abstract}

DOI: 10.1103/PhysRevLett.94.193902

PACS numbers: 42.25.Bs, 42.79.Ag

Ebbesen et al. [1] reported the observation of enhanced transmission of light through subwavelength holes in an otherwise optically opaque metal film. This observation has stimulated a large body of research ([2-7], and references therein) into resonantly enhanced transmission through metal apertures. It is known that for a given wavelength there are no modes supported by holes below a certain diameter in a perfect conductor [8]. (This of course is not the situation in the studies by Ebbesen and colleagues where the finite conductance of the metal may provide complex mode solutions even when real mode solutions are forbidden.) By contrast, a slit supports a set of strongly resonant Fabry-Perot-like TEM modes for a metal plate which is at least half the wavelength thick. This has been confirmed by a range of experiments and theoretical studies [4,6,9-15], where in addition, even for metals at microwave frequencies, it has been shown that as the slit width is reduced to very small values the resonant frequency may be substantially reduced due to the skin effect distorting the fields in the slit [16]. The question arises as to what happens if the slit is bent into a circle, forming an annulus. Now the condition that prohibits the existence of modes below a certain radius is no longer true and the annulus will support TEM modes as well as other modes found in a coaxial waveguide.

Recently, Badia and Van Labeke [17] and Moreau et al. [18] proposed, through numerical simulation, that an array of such apertures would exhibit a substantial increase in transmission efficiency compared to hole arrays. This was later confirmed experimentally at near infrared wavelengths by Fan et al. [19]. In the present study, however, we explore for the first time the resonant transmission of microwaves through a single annular aperture in a thick metal plate. Fabry-Perot-like modes with resonant frequencies quantized by the cavity length $L$ may be supported. Further, the arrangement will support modes which are additionally quantized by the circumference of the annulus. Consider the sample shown in the inset of Fig. 1.

Assuming the total wave vector within the metal cavity is the same as that in free space (because the metal is a near perfect conductor), and ignoring end effects, we may write the resonant frequencies approximately as

$$
f_{(P, N)}=\sqrt{\left(\frac{2 P}{a+b}\right)^{2}+\left(\frac{N \pi}{L}\right)^{2}} \frac{c}{2 \pi},
$$

where $P$ is the azimuthal mode number (half the number of nodes) around the circumference of the annulus, $a$ is the radius of the inner core, $b$ is the outer radius, and $N$ is the longitudinal mode number. This simple analysis does not take into account the radial nature of the electric $(E)$ field within the space bounded by the two concentric cylinders, but assumes a uniform field as in the case of a twodimensional slit. This proves to be a good approximation when $a$ is comparable in magnitude to $b$. A more rigorous treatment of this geometry would require a Bessel function of the first kind to fully describe the field $[8,20]$.

It will be shown that it is indeed possible to couple incident radiation to families of $P$ modes each containing multiple values of $N$. Most significantly, modes with $P=0$ are supported, which are essentially just the infinite slit modes, but these are excited only with radiation incident off normal.

The sample (Fig. 1, inset) consists of a cylindrical cavity of radius $b=1.50 \mathrm{~mm}$ drilled into an aluminum alloy substrate of dimensions $600 \times 600 \times 30 \mathrm{~mm}$, thus forming an air-filled cylindrical cavity of length $L=$ $30.00 \mathrm{~mm}$. A solid cylindrical core of the same material but slightly smaller radius $(a=1.25 \mathrm{~mm})$ is inserted into the hole to form a coaxial arrangement with a subwavelength gap width of $0.25 \mathrm{~mm}$. The inner core is held in situ by attaching a $0.08 \mathrm{~mm}$ diameter glass fiber radially across the substrate and inner core on both the incident and exit 


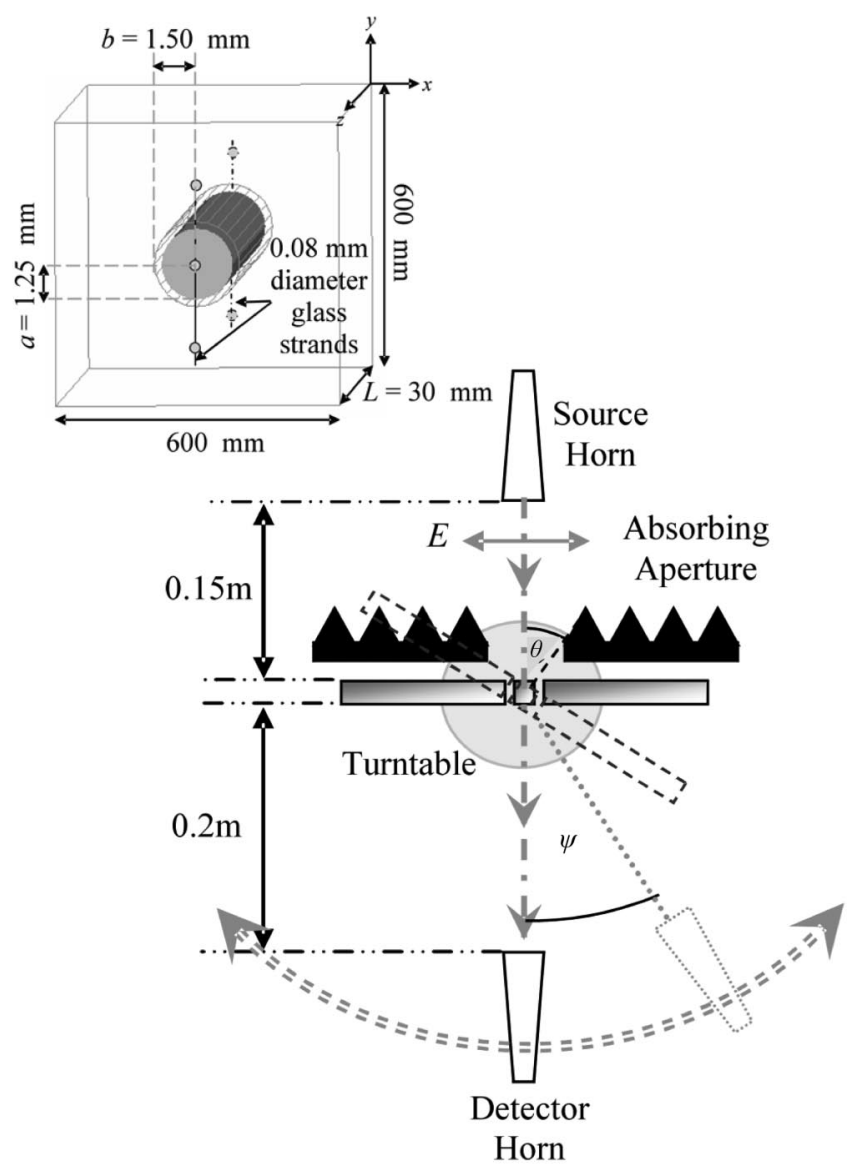

FIG. 1. Experimental arrangement: Matched standard-gain source and detector horns are connected to a sweep oscillator and scalar network analyzer, respectively. Inset: Sample geometry (see text).

in-plane surfaces of the sample. Each fiber was orientated at $90^{\circ}$ to the plane containing the incident electric vector to minimize electromagnetic disturbance. To record transmission spectra the sample was arranged as shown in Fig. 1. The source horn antenna was positioned at a distance of $0.20 \mathrm{~m}$ from the sample to provide approximately plane wave incident radiation. The sample was placed on a turntable with the detector horn mounted upon an arm extending from this turntable, and positioned $0.15 \mathrm{~m}$ from the sample. While the source remained fixed, the sample could be rotated to chosen values of $\theta$. For each incident angle, the detector scanned the transmission angle $\psi$ within the range $-70^{\circ}<\psi<70^{\circ}$ with a resolution of $0.2^{\circ}$. We define $\psi=0^{\circ}$ as normal to the surface plane of the sample as shown by Fig. 1. Configuring the experimental setup in this way allowed the angular distribution of the transmitted signal in the plane of polarization to be recorded as a function of frequency, for values of $\theta$ in the range $0<\theta<70^{\circ}$, in increments of $10^{\circ}$.

The inset of Fig. 2 shows the transmission spectrum obtained when illuminating the annulus arrangement with plane wave radiation at normal incidence. The detector is

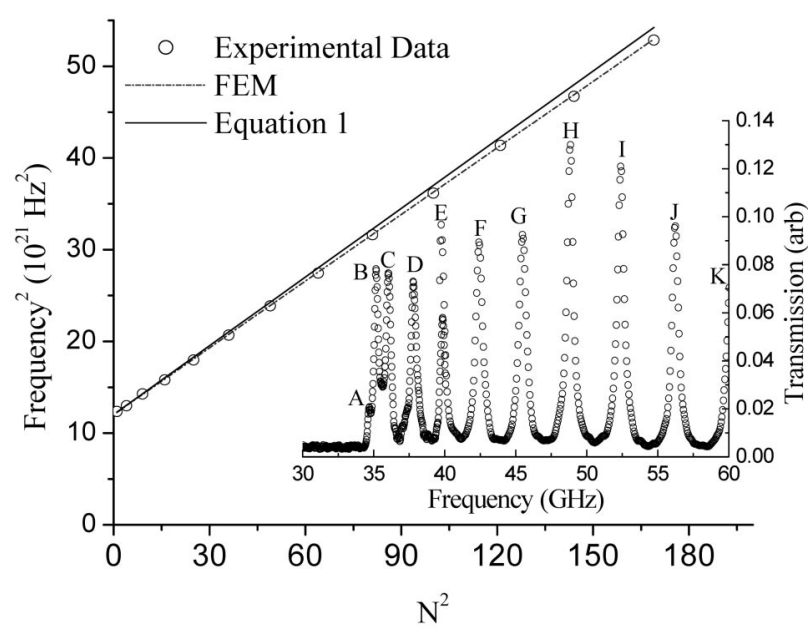

FIG. 2. Resonant frequency squared as a function of $N^{2}$ for the experimental transmission data obtained at $\theta=0^{\circ}, \psi=0^{\circ}$, compared to that calculated using the FEM code. Also shown are the predictions of Eq. (1), generated using consecutive values of $N$ between 1 and 13, and a circumferential quantum number of 1. Inset: The transmission spectrum obtained at $\theta=0, \psi=$ $0^{\circ}$. A total of 11 resonant modes are clearly visible over the selected frequency range.

positioned normal to the exit face of the sample at $\psi=0^{\circ}$. A total of 11 peak transmission intensities labeled A through $\mathrm{K}$, which occur at frequencies corresponding to resonant guided modes of the system, are clearly visible over the selected frequency range. The slightly irregular variation of the relative intensities of the modes is associated with the difference in detection efficiency between the detector horns used for the $26.5-40 \mathrm{GHz}$ and $40-60 \mathrm{GHz}$ ranges.

The simple analysis of the frequency of the modes of the system [Eq. (1)] predicts that the square of the resonant frequency is proportional to $N^{2}$. The graph shown in Fig. 2 clearly demonstrates that this is a good approximation. Also shown are the predictions of the resonant frequencies from the finite element method (FEM) modeling that agree exceptionally well with the experimental data. However, note that, for the shorter wavelengths, the predictions from Eq. (1) deviate from both the experimental results and from the FEM model [e.g., for $N=10$, Eq. (1) predicts the mode to be $0.7 \mathrm{GHz}$ higher in frequency than observed experimentally]. This discrepancy is associated with a Takakura-type [10] shift that arises simply from end effects. FEM modeling shows that these modes are $P=1$ modes [the $P=1, N=2$ mode field profiles are shown in Fig. 3(a)]. Mode A, being the $N=0$ mode [Fig. 3(b)], has an almost dc character along the length of the annulus and is clearly not so strong as the $N>0$ modes. The intensity of this particular mode is influenced more by the finite conductance of the metal. A FEM model of the response with a perfect conductor predicts the transmitted intensity 


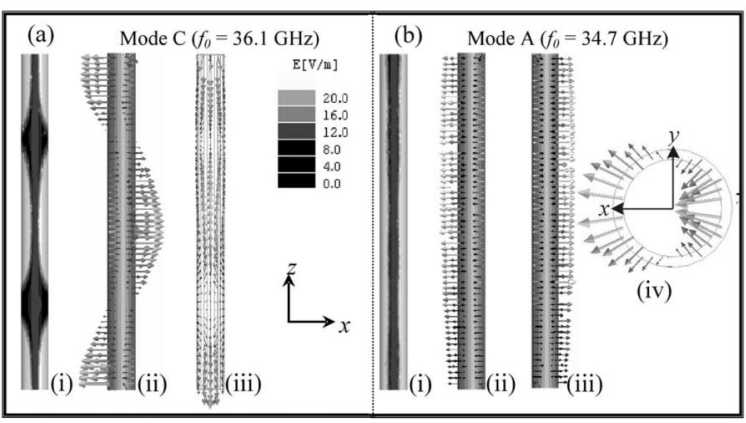

FIG. 3. The time averaged magnitude [(i)] of the electric field together with the $E$ - and $H$-vector magnitudes [(ii) and (iii), respectively], evaluated at the peak transmission frequency of (a) mode C (36.1 GHz) and (b) mode A (34.7 GHz). The $E$ - and $H$-vectors are calculated at values of phase corresponding to maximum intensity. Plane wave radiation is incident from above with its wave vector in the $z$ direction, and incident electric vector lying parallel to the $x$ axis. Also shown (iv) is the electric vector magnitude calculated at the resonant frequency of mode A over the $x y$ plane at $z=0$ (the illuminated face of the sample).

due to this mode to be much nearer than that of the higher order modes.

For all $P=1$ and higher odd-numbered modes, coupling by plane wave radiation is possible at normal incidence since the electric vectors on either side of the inner core must oscillate in phase. By contrast, incident radiation may only be coupled to modes corresponding to even values of azimuthal quantum number $(P)$ at $\theta \neq 0^{\circ}$, since a phase variation of the $E$ vector, in the projected direction of that vector on the surface plane, must be introduced across the annulus.

Figure 4 shows transmission as a function of frequency for four values of $\theta$ in $20^{\circ}$ increments. Each transmission spectrum is taken with the detector at $\psi=60^{\circ}$. Modes $\mathrm{B}$, $\mathrm{C}, \mathrm{D}$, and $\mathrm{E}$ reduce in intensity as the angle of incidence is increased. This is as expected since these modes are all $P=1$ modes which become more difficult to excite as the horizontal phase variation across the annulus increases. By contrast, modes $a^{*}$ and $b^{*}$, which are not visible for normal incidence, show increased coupling with increasing $\theta$. It is apparent that these two modes, one of which is lower in frequency than the cutoff frequency for the TE mode, have symmetry requirements that are incompatible with a driving incident plane wave at normal incidence. These are both $P=0$ modes, and are essentially infinite slit modes, quantized in the axial direction only.

The field profiles of mode $a^{*}(34.1 \mathrm{GHz})$ are plotted over the cylindrical inner core in Fig. 5. In this case, radiation is incident in the $x z$ plane, at an angle of $60^{\circ}$ from normal to the illuminated face of the sample and TM polarized (i.e., the $E$ vector also lying in the $x z$ plane). The time averaged $E$-field magnitude [Fig. 5 (i)] shows mode $a^{*}$ to be a standing wave state in $z$, with mode numbers $P=0, N=$ 7. Note here that the distortion in the time averaged $E$-field

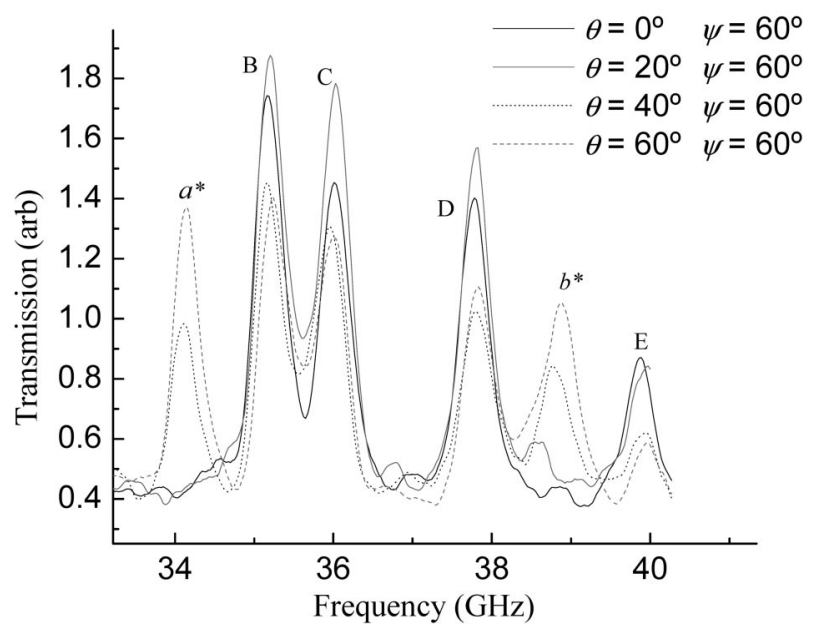

FIG. 4. Frequency dependant transmission spectra obtained for four consecutive values of $\theta$ in the range $0<\theta<60^{\circ}$, in increments of $20^{\circ}$. Each transmission spectrum is taken at a transmission angle $\psi=60^{\circ}$.

magnitude, which is more pronounced at the illuminated end of the structure, is due to the relatively large angle of incidence. The $E$-vector magnitude [Fig. 5 (ii) and (iii) plotted half a cycle apart] and magnetic $(H)$-vector magnitude [Fig. 5 (iv) at a phase corresponding to maximum field] reveal the nature of the mode. Because of the azimuthal symmetry of the coaxial structure, there is no field

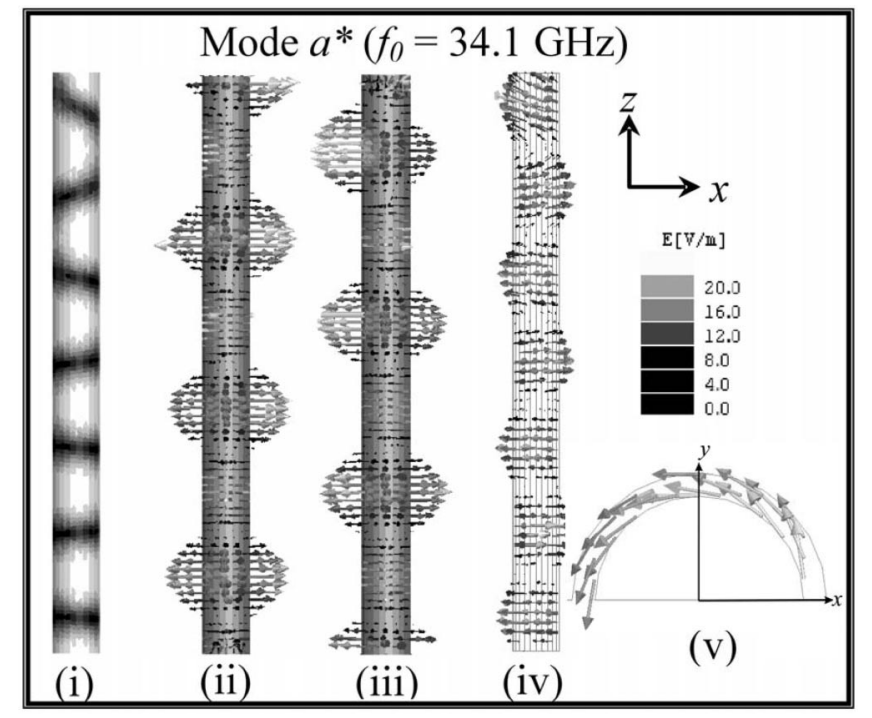

FIG. 5. The field profiles calculated using the FEM code at a fixed frequency of $34.1 \mathrm{GHz}$, corresponding to the resonant frequency of mode $a^{*}$, with $\theta=60^{\circ}, \psi=60^{\circ}$. (i) The time averaged electric field magnitude. (ii) and (iii) $E$-vector magnitudes evaluated at phases separated by $180^{\circ}$. (iv) The $H$-vector magnitude at a phase corresponding to maximum field. (v) The $H$-vector magnitude calculated over a plane parallel to the $x y$ plane at $z=-15 \mathrm{~mm}$, at a phase corresponding to maximum field. 


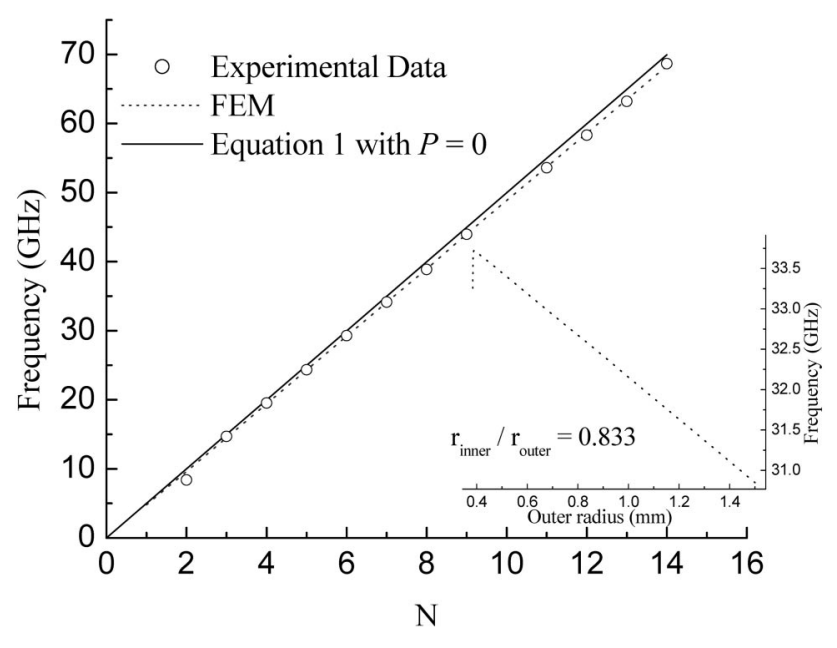

FIG. 6. Resonant frequency as a function of $N$ for the experimental transmission data obtained at $\theta=60^{\circ}, \psi=60^{\circ}$, compared to the modeled response of the sample calculated using the FEM code. Also shown are the predictions of Eq. (1). Inset: Modeled resonant frequency as a function of outer radius of the annulus for the $P=0, N=1$ mode. In this instance, a $4.29 \mathrm{~mm}$ thick substrate is used, and the inner to outer radius ratio remains constant.

variation in $\phi$. The $E$ vector within the cavity is shown to be radial, oscillating with time entirely in the $x z$ plane. The magnetic vector is orthogonal to the $E$ vector, containing only $H_{x}$ and $H_{y}$ components. Mode $a^{*}$ is a TEM mode having a propagation constant $\beta$ and wave impedance $Z$ equal to the wave number $k_{0}$ and wave impedance $Z_{0}$ of a plane wave propagating in free space. The cutoff wave number $k_{c}=\sqrt{k_{0}^{2}-\beta}$ is zero, and $\lambda_{\phi}=\infty$. To first order the resonant frequency is dependent only upon the length of the annulus and independent of its radius. A plot of frequency against mode number for all the readily observed modes of the $P=0$ family is shown in Fig. 6 .

Having found this family of azimuthally independent modes, it is worthwhile exploring how the resonant frequency varies with the overall size of the annulus, keeping the inner to outer radius ratio constant. This modeling has been undertaken for a thinner plate supporting the $P=0$, $N=1$ mode and is illustrated in the inset of Fig. 6. There are three points to note. First, the frequency increases slightly as the radius is reduced due to diminution of end effects. Second, the frequency eventually starts to fall as the thin gap becomes less than about $70 \mu \mathrm{m}$ (the PreistSambles effect due to finite conductance [16]). Third, the mode broadens as its frequency reduces, and the transmitted signal diminishes substantially. More importantly of course, it suggests that extremely small annuli in thin metal plates will transmit microwaves resonantly via TEM modes.

In conclusion, we have recorded resonant transmission of microwaves through a single small annular slit in a metal plate. At normal incidence a family of modes with azimuthal quantum number 1 are found, with frequencies dictated by the plate thickness and the mean radius of the annulus. For non-normal incidence a second family of modes is found corresponding to zero azimuthal quantum number. These modes, essentially like those of an infinite slit, have frequencies dictated by the plate thickness and, were it not for the finite conductance of the plate, would allow annular transmission at negligible radius. This shows that the enhanced hole transmission recorded by Ebbesen and co-workers may now be substantially increased by putting a central metal post in each hole and ensuring the pitch of the grating and the metal film thickness are in accord.

This work was carried out as part of the Emerging and Underpinning Technologies Domain of the MOD Research Program.

[1] T. W. Ebbesen et al., Nature (London) 391, 667 (1998).

[2] H. F. Gheami et al., Phys. Rev. B 58, 6779 (1998).

[3] T. Lopez-Rios et al., Phys. Rev. Lett. 81, 665 (1998).

[4] J. A. Porto, F. J. Garcia-Vidal, and J. B. Pendry, Phys. Rev. Lett. 83, 2845 (1999).

[5] E. Popov et al., Phys. Rev. B 62, 16100 (2000).

[6] S. Astilean, P. Lalanne, and M. Palamaru, Opt. Commun. 175, 265 (2000).

[7] L. Martin-Moreno et al., Phys. Rev. Lett. 86, 1114 (2001).

[8] J.A. Stratton, Electromagnetic Theory (McGraw-Hill, New York, 1941).

[9] H. E. Went et al., Appl. Phys. Lett. 77, 2789 (2000).

[10] Y. Takakura, Phys. Rev. Lett. 86, 5601 (2001).

[11] F. Yang and J. R. Sambles, Phys. Rev. Lett. 89, 063901 (2002).

[12] A. P. Hibbins, J. R. Sambles, and C. R. Lawrence, Appl. Phys. Lett. 81, 4661 (2002).

[13] P. Stavrinou and L. Solymar, Opt. Commun. 206, 217 (2002).

[14] H. J. Lezec et al., Science 297, 820 (2002).

[15] L. Martin-Moreno et al., Phys. Rev. Lett. 90, 167401 (2003).

[16] J. R. Suckling et al., Phys. Rev. Lett. 92, 147401 (2004).

[17] F. I. Baida and D. Van Labeke, Opt. Commun. 209, 17 (2002).

[18] A. Moreau et al., Opt. Express 11, 1131 (2003).

[19] W. Fan et al., Phys. Rev. Lett. 94, 033902 (2005).

[20] R. E. Collin, Field Theory of Guided Waves (IEEE, New York, 1991), 2nd ed. 